TIPA. Travaux interdisciplinaires sur la parole et le langage

28 | 2012

Linguistique expérimentale

\title{
Étude expérimentale de la perception de la modalité à travers l'intonation en anglais
}

Jean-Marie Merle et Peter Prince

\section{CpenEdition}

Journals

Édition électronique

URL : http://journals.openedition.org/tipa/202

DOI : 10.4000/tipa.202

ISSN : 2264-7082

Éditeur

Laboratoire Parole et Langage

Référence électronique

Jean-Marie Merle et Peter Prince, «Étude expérimentale de la perception de la modalité à travers l'intonation en anglais », TIPA. Travaux interdisciplinaires sur la parole et le langage [En ligne], 28 | 2012, mis en ligne le 29 octobre 2012, consulté le 19 avril 2019. URL : http://journals.openedition.org/ tipa/202 ; DOI : 10.4000/tipa.202

Ce document a été généré automatiquement le 19 avril 2019

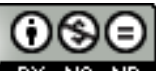

La revue TIPA. Travaux interdisciplinaires sur la parole et le langage est mise à disposition selon les termes de la licence Creative Commons Attribution - Pas d'Utilisation Commerciale - Pas de Modification 4.0 International. 


\section{Étude expérimentale de la perception de la modalité à travers l'intonation en anglais}

Jean-Marie Merle et Peter Prince

\section{Introduction ${ }^{1}$ - les questions bipolaires}

1 En français, on peut former une question fermée - également appelée question totale, ou bipolaire, c'est-à-dire appelant une réponse positive ou négative - soit en mobilisant une syntaxe adéquate (par exemple : « Est-ce que tu sais nager ?» ou « Sais-tu nager ? »), soit en gardant la syntaxe d'un énoncé déclaratif mais en utilisant une intonation interrogative, marquée graphiquement par la seule présence d'un point d'interrogation : «Tu sais nager?» Dans les interactions quotidiennes, cette dernière façon de formuler une question fermée est de loin la plus utilisée ${ }^{2}$.

2 Ce même phénomène existe dans bien d'autres langues mais ne s'emploie pas avec la même fréquence ou selon les mêmes conditions d'usage. En anglais on y a moins souvent recours, l'inversion entre sujet et verbe étant la façon usuelle de former une question. Ainsi un anglophone dira plus facilement 'Can you swim ?' que 'You can swim ?' Alors qu'en français cette simple transformation de l'intonation en modalité interrogative peut s'employer pour initier une conversation, sans que d'autres données pragmatiques ou linguistiques soient nécessaires, en anglais ce n'est pas le cas. Gunlogson (2001) note que de tels énoncés à structure déclarative et à intonation ascendante ne peuvent pas venir de but en blanc ("out of the blue»), mais requièrent des informations linguistiques ou visuelles préalables que le locuteur reprend, explicitement ou implicitement, en formulant son énoncé. Ainsi 'You can swim?' ne sera produit que si le contexte linguistique ou visuel a permis au locuteur de constater que son interlocuteur sait nager. Il ne s'agit pas ici d'une recherche d'information, mais d'une demande de confirmation. Pour Gunlogson (2001), il s'agit plutôt d'une remise à jour (update) du contexte de la part 
du locuteur. D'autres déclaratifs ascendants peuvent être de véritables questions, mais nécessitent néanmoins un contexte préalable.

Une deuxième caractéristique, moins systématique mais néanmoins fréquente, de ce type de question en anglais est qu'elle peut être assortie d'une intention communicative particulière, ce qui confère un caractère plus marqué à l'intonation. Par exemple 'You can swim?' en anglais serait plutôt l'expression d'un doute ou d'une surprise qu'une recherche d'information; ou, si l'accentuation se plaçait sur l'auxiliaire, ce serait une demande de confirmation. Dans ce cas, l'énoncé produit pourrait également se terminer par le tag 'can't you?'.

Bien que moins fréquent en anglais qu'en français, ce phénomène est largement attesté (Armstrong \& Ward, 1926; O'Connor \& Arnold, 1961; Wells, 2006) et se produit assez souvent pour qu'un étudiant en anglais langue seconde (L2) y soit confronté régulièrement, ce qui sollicite une aptitude à distinguer des questions de ce type afin de communiquer. Suivant Faraco \& Cavé (2008) qui ont examiné cette question pour le français, nous avons effectué une expérience portant sur la perception des énoncés de ce type en anglais en adoptant le même protocole expérimental qu'eux.

\section{Matériau : constitution du corpus, enregistrement et découpage}

5 Trente énoncés ont donné lieu chacun à un enregistrement, sous deux formes intonatives différentes (interrogative et déclarative), et ont été réalisés par deux locuteurs anglophones (un homme et une femme). Ces énoncés étaient répartis en trois séries.

- La première série était constituée de dix énoncés à la syntaxe canonique (comportant un sujet et un prédicat verbal);

- la deuxième série était constituée de dix énoncés syntaxiquement tronqués ;

- la troisième série comportait des difficultés d'ordre lexical.

Les interrogatives des deux premières séries, ainsi que leurs contextes linguistiques immédiats, proviennent des dialogues d'un roman de Lisa Gardner³. Pour la version déclarative de ces mêmes énoncés, un contexte linguistique a été ajouté pour leur donner plus d'authenticité. Les énoncés de la troisième série ont été inventés et comprennent des mots et des expressions rares peu susceptibles d'être connus des francophones. Pour que les énoncés interrogatifs de cette troisième série respectent les contraintes pesant sur la production de tels énoncés en anglais, un contexte a été inventé et ajouté. Les énoncés déclaratifs de cette même série n'obéissant pas aux mêmes contraintes, il n'était dès lors pas nécessaire de les mettre en contexte.

7 Ces trente énoncés ont ainsi généré 120 items diffusés dans un ordre rendu aléatoire par le logiciel Perceval (Cavé et al., 1998; André et al., 2003). Pour que l'enregistrement, effectué dans les studios du LPL, soit réalisé avec une intonation authentique, les deux locuteurs, un homme et une femme, l'un et l'autre anglophones natifs, lisaient non seulement les énoncés mais aussi les contextes afin de pouvoir sans hésiter réaliser l'intonation attendue. Par exemple, le segment 1.3 ci-dessous - You're all right, Kimberly reçoit successivement un contexte amenant une intonation interrogative, puis un contexte amenant une intonation déclarative : 
She grabbed the back of a chair. Her face looked grey.

"You're all right, Kimberly?" (SG 262)

$9 \quad 1.3$ déclaratif

"You've got a job, a family. You're all right, Kimberly. It's Sal I'm worried about."

Pendant les séances d'enregistrement, les deux expérimentateurs ont écouté les locuteurs et leur ont demandé de reprendre toute phrase produite avec une erreur de prononciation ou d'intonation. Après l'enregistrement, les segments ont été découpés de façon à ce que les participants, lors de l'expérience, n'entendent que l'énoncé interrogatif ou déclaratif (segments en italiques dans l'exemple donné ci-dessus).

\section{Participants}

11 Les participants se sont prêtés à l'expérience sur la base du volontariat. Les non anglophones étaient tous des étudiants de l'Université de Provence. Il y avait 24 participants dans chaque groupe et la moyenne d'âge des trois groupes se situait entre 21 et 28 ans.

12 Le groupe des non anglicistes comportait 17 femmes et 7 hommes. Ces étudiants étaient en $3^{\text {ème }}$ année, soit en arts plastiques, soit en lettres modernes, soit en psychologie. Ils étudiaient l'anglais en moyenne depuis 10 ans et au moment de l'expérience continuaient à l'étudier à raison de deux ou trois heures par semaine. Aucun de ces étudiants n'avait séjourné en pays anglophone pour une période de plus d'un mois.

13 Le groupe des anglicistes était constitué de 21 femmes et de 3 hommes. Ils étudiaient l'anglais depuis 11 ans en moyenne, mais de manière plus intensive à l'université que les non anglicistes. Ils avaient tous passé de 9 à 12 mois en pays anglophone.

14 Le groupe des anglophones était composé d'une part de lecteurs ou d'enseignants à l'Université de Provence, de nationalité britannique ou américaine, et d'autre part d'étudiants venus des États-Unis pour les cours d'été de l'université. Le groupe d'anglophones comportait 14 femmes et 10 hommes.

\section{Procédure}

Une fois les 120 énoncés découpés et regroupés, l'expérience décrite ici a été réalisée dans les conditions suivantes.

Chaque participant était placé devant un boîtier comportant deux boutons, une main sur chacun des boutons, prêt à réagir aux stimuli. Derrière le boîtier un écran rappelait, d'un bout à l'autre de l'expérience, sur quel bouton appuyer lorsque l'énoncé entendu était interrogatif, et sur quel bouton appuyer lorsque l'énoncé était déclaratif.

Pour éviter les perturbations, l'écoute se faisait à l'aide d'un casque. Chaque participant avait reçu pour consigne de réagir aussi vite que possible, tout en faisant le moins d'erreurs possible. Le temps alloué pour répondre était de trois secondes. Il y avait six items d'entraînement pour familiariser les participants avec la tâche. Ces items n'ont pas été pris en compte lors de l'analyse statistique.

Le logiciel Perceval a permis ensuite de mesurer et d'enregistrer le temps de réponse de chacun des 72 participants à l'écoute de chacun des énoncés, tout en précisant quels 
items avaient donné lieu à des erreurs et quels autres avaient donné lieu à de bonnes réponses.

\section{Caractéristiques des énoncés} sont néanmoins variées :

1. Syntaxe minimale sujet + auxiliaire (prédicat elliptique) :

She did? / She did.

2. SN objet à déterminant explicite :

You got an address? / You got an address.

3. Attribut + apostrophe :

You're all right, Kimberly? / You're all right, Kimberly.

4. Énoncé intransitive :

You'll call? / You'll call.

5. Syntagme nominal objet à déterminant zéro :

We have vegetables? / We have vegetables.

6. Syntagme nominal objet (déterminant + Adj + noyau nominal) + apostrophe :

You want some extra money, son? / You want some extra money, son.

7. Pronom objet :

You know what? / You know what.

8. 2 syntagmes nominaux objets :

He got a real name, a physical address? / He got a real name, a physical address.

9. Syntagme nominal objet et accent sur le sujet :

You delivered them? / You delivered them.

10. Sujet et prédicat attribut locatif :

He's out of town? / He's out of town.

\subsection{Deuxième série d'énoncés}

Les énoncés de la deuxième série ne comportaient pas d'assemblage prédicatif, ce qui en soi constitue une difficulté sur le plan syntaxique puisque ce sont des phrases tronquées. Les quatre premiers étaient constitués de syntagmes nominaux, le cinquième d'un syntagme adjectival, les cinq derniers d'un prédicat sans sujet exprimé.

TIPA. Travaux interdisciplinaires sur la parole et le langage, 28 | 2012 
Ces énoncés tronqués ont eux aussi des caractéristiques diverses

1. Syntagme nominal incluant une coordination:

Boy or girl? / Boy or girl.

2. Nom composé :

Socioeconomics? / Socioeconomics.

3. Nom dérivé :

Hunter? / Hunter.

4. Syntagme nominal: Adjectif + Nom :

Tough case? / Tough case.

5. Syntagme adjectival :

Scared? / Scared.

6. Verbe + Pronom :

Got it? / Got it.

7. Verbe en -ing intransitif :

Working? / Working.

8. Verbe + Nom :

Got a microscope? / Got a microscope.

9. Verbe + complément infinitif :

Wanna touch it? / Wanna touch it.

10. Verbe + enchâssement à mode fini :

Think she'll do it? / Think she'll do it.

\subsection{Troisième série d'énoncés}

Les énoncés de la troisième série étaient syntaxiquement complets mais comportaient des difficultés lexicales, mots et locutions rares de fréquence d'emploi très basse: 1,6 occurrences par million (Davies, 2011). Les énoncés de cette série ont eux aussi une diversité mise en évidence ci-après :

1. Construction transitive de valence $2+$ prétérit :

He used a winch? / He used a winch.

2. Copule + prétérit + syntagme nominal :

It was just a lark? / It was just a lark.

3. Copule + présent + adjectif participial :

He's released on bail? / He's released on bail.

4. Forme be + Ving + présent :

They're making a fuss? / They're making a fuss.

5. Locution transitive de valence 3 + prétérit :

She took a swipe at him? / She took a swipe at him.

6. Construction transitive faible + présent :

She wears an apron? / She wears an apron.

7. Construction intransitive au prétérit + particule adverbiale :

They touted around? / They touted around.

8. Construction intransitive au présent + co-prédicat busking :

He goes busking? / He goes busking.

9. Copule + présent + syntagme prépositionnel attribut locatif :

It's in the hutch? / It's in the hutch.

10. Enchâssement d'une subordonnée à mode fini : 
You reckon it's naff? / You reckon it's naff.

\section{Résultats}

Chaque participant a réagi à l'écoute des 120 items, ce qui donne un total de 2880 réponses par groupe et de 8640 réponses pour l'ensemble des trois groupes. Les réponses erronées ou l'absence de réponse, c'est-à-dire les cas où le participant a laissé passer le délai de latence maximal fixé à trois secondes sans fournir de réponse, n'ont pas été prises en compte dans le traitement statistique des temps de réponse. Dans la mesure où l'on cherche à déterminer avec quelle rapidité les participants reconnaissent la modalité des énoncés, il est difficile en effet d'intégrer aux statistiques les cas où la modalité n'est pas reconnue.

L'analyse statistique des taux d'erreurs révèle toutefois des effets significatifs en fonction du groupe $(F(2,69)=4,64, p<.05)$, et du type d'énoncés $(F(2,138)=86,94, p<0,001)$. Comme indiqué dans le tableau 1, l'effet de groupe se manifeste par un nombre croissant d'erreurs lorsque le niveau de compétence baisse.

Quant aux types d'énoncés, la série 3 (énoncés comportant une difficulté lexicale) a donné la plus faible proportion d'erreurs (5\%), suivie de la série 1 (énoncés syntaxiquement complets) avec $11 \%$ d'erreurs, tandis que la série 2 (énoncés syntaxiquement tronqués) a donné lieu à la plus forte proportion d'erreurs: 15,5\%. Ces statistiques sur le taux d'erreurs reflètent très fidèlement celles concernant les temps de réponse. Ainsi les deux types de données sont complémentaires l'un de l'autre.

Tab. 1. Non-réponses et erreurs pour les trois groupes

\begin{tabular}{|l|l|l|l|}
\hline & Non-Réponses & Erreurs & $\%$ du total \\
\hline Non anglicistes & 31 & 343 & 12,99 \\
\hline Anglicistes & 36 & 285 & 11,15 \\
\hline Anglophones & 26 & 211 & 8,23 \\
\hline
\end{tabular}

Les réponses correctes mais anticipées, c'est-à-dire lorsque le participant répond avant la fin de l'énoncé, ont été gardées. Il est possible en effet de repérer une intonation avant la fin d'un énoncé et de répondre en conséquence. Ces réponses anticipées sont au nombre de $124 \mathrm{chez}$ les non anglicistes ( $4.31 \%$ du total), $228 \mathrm{chez}$ les anglicistes $(7,92 \%)$ et 329 chez les anglophones (11,42\%).

Des analyses de variance ont été appliquées pour traiter les données concernant les temps de réponse. Il en ressort que l'on peut dégager des tendances propres à chaque groupe ( $\mathrm{F}$ $(2,69)=3,44, \mathrm{p}<.05)$ :

- les non anglicistes ont les temps de réponse les plus longs ;

- les anglophones, les temps de réponse les plus courts.

La moyenne des temps de réponse est de 619 ms (écart type $=294,4$ ) pour les non anglicistes, de 525,8 ms (écart type $=331,9$ ) pour les anglicistes et de 427,6 ms (écart type $=309,1)$ pour les anglophones. 
Globalement, la moyenne des temps de réponse aux deux modalités, déclarative et interrogative, ne donne pas de différence significative $(p=0,51)$. On trouve cependant une interaction entre groupe et modalité $(F(2,69)=6,83, p<.01)$, telle que les temps de réponse sont plus longs pour les énoncés interrogatifs que pour les énoncés déclaratifs chez les deux groupes non anglophones, alors que cette situation s'inverse chez les locuteurs anglophones (voir figure 1).

Fig. 1. Temps de réponse moyen (en $\mathrm{ms}$ ) pour les trois groupes et les deux modalités

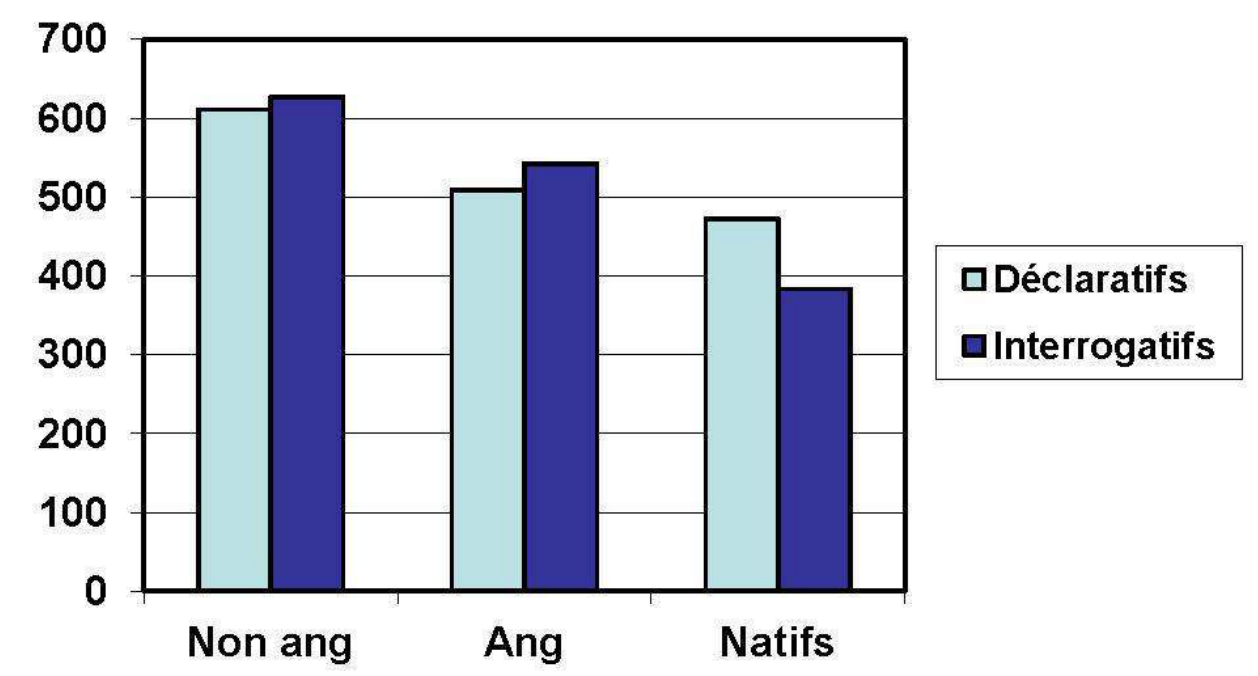

32 Les types d'énoncés ont également un effet non négligeable sur les temps de réponse (F $(2), 138)=18,59, p<.001)$. La moyenne des temps de réponse est sensiblement la même pour les énoncés de la $1^{\text {ère }}$ série, syntaxiquement complets, et les énoncés de la $3{ }^{\text {ème }}$ série, syntaxiquement complets mais comportant une difficulté lexicale (respectivement 501,7 ms et 506,0 ms en moyenne), tandis que le temps de réponse pour les énoncés de la 2 ème série, syntaxiquement incomplets, est significativement plus long (575,6 ms en moyenne).

Globalement les participants ont répondu $100 \mathrm{~ms}$ plus rapidement à la voix masculine qu'à la voix féminine (478 ms contre $577 \mathrm{~ms}$ ). Ce résultat est l'inverse de celui trouvé par Faraco \& Cavé (2008) : il est sans doute lié aux caractéristiques propres à la lecture de chacun des locuteurs lors de l'enregistrement.

Enfin on trouve une interaction entre modalité et type d'énoncés $(F(2,138=62,55$, $\mathrm{p}<.0001$ ). Pour les deux premiers types d'énoncés (énoncés syntaxiquement complets et énoncés syntaxiquement incomplets) le temps de réponse est plus long pour la modalité déclarative que pour la modalité interrogative, alors que la situation s'inverse nettement pour les énoncés syntaxiquement complets mais comportant une difficulté lexicale, ceux de la troisième série, pour lesquels le temps de réponse est plus long pour la modalité interrogative que pour la modalité déclarative (voir tableau 2). 
Tab. 2. Temps de réponse, tous groupes confondus, aux trois types d'énoncés dans les deux modalités

\begin{tabular}{|l|l|l|l|l|}
\hline & \multicolumn{2}{|l|}{ Énoncés déclaratifs } & \multicolumn{2}{l|}{\begin{tabular}{l}
\multicolumn{2}{l|}{ ínoncés } \\
interrogatifs
\end{tabular}} \\
\cline { 2 - 5 } & Moyenne & Écart type & Moyenne & Écart type \\
\hline Syntaxe complète & 561,7 & 345,9 & 441,6 & 357,2 \\
\hline Syntaxe incomplète & 591,9 & 304,4 & 559,2 & 310,1 \\
\hline Difficulté lexicale & 434,8 & 264,7 & 577,3 & 321,7 \\
\hline
\end{tabular}

\section{Discussion}

\subsection{Gradation des résultats - l'hypothèse du contact}

Conformément à ce que l'on pourrait penser, le niveau des participants influence leur capacité à distinguer rapidement un énoncé interrogatif d'un énoncé déclaratif avec comme seul indice l'intonation. En d'autres termes, les anglophones sont plus sensibles à ce paramètre et lui attribuent plus rapidement l'interprétation appropriée.

Si la performance est moins bonne chez les apprenants, le contact prolongé avec l'anglais parlé à travers l'immersion dans un pays anglophone mène néanmoins à une sensibilité accrue à ce phénomène intonatif. Chez les non spécialistes, pour qui le contact avec l'anglais parlé se limite essentiellement à l'écoute d'un enseignant ou d'enregistrements en cours, l'aptitude à percevoir l'intonation comme indice permettant de discerner la modalité est moindre. Cette moins bonne performance chez les francophones se reflète également dans les taux d'erreurs, qui sont plus élevés pour ces deux groupes, mais pas de manière considérable (cf. tableau 1).

Globalement la tâche est réalisable: les non anglicistes réussissent à répondre correctement dans $87 \%$ des cas. On peut donc dire que l'information fournie par l'intonation est tout à fait perceptible et interprétable par les deux groupes d'apprenants, mais moins ils ont été exposés à l'anglais parlé, plus ils ont besoin de temps pour traiter cette information. Un séjour prolongé en pays anglophone accroît sans doute la sensibilité d'un francophone à de nombreux phénomènes intonatifs, dont celui-ci.

\subsection{Familiarité - pour les francophones - du phénomène de l'interrogation sans syntaxe interrogative}

Que la tâche soit réalisable par des francophones avec un taux d'erreurs relativement bas peut s'expliquer d'abord par la fréquence avec laquelle les francophones utilisent l'intonation comme seul indice pour distinguer la modalité dans leur propre langue. Même si le phénomène est moins fréquent en anglais, un transfert positif de cette habitude du français vers l'anglais favorise vraisemblablement le discernement de la modalité. Par ailleurs, les contours intonatifs de l'anglais font généralement l'objet de 
quelques séances d'entraînement dans le secondaire, ce qui sensibilise également les apprenants. Ces raisons font que la tâche est à la portée de tous et que l'absence d'indices grammaticaux canoniques propres à la forme interrogative n'a pas perturbé les francophones outre mesure.

Il serait intéressant de mener cette même étude auprès d'étudiants en anglais faisant leurs études dans d'autres pays, où les conditions d'emploi de l'intonation comme manière de former une question sont différentes, et d'examiner la question de plus près en incluant des stimuli syntaxiques canoniques, ce qui permettrait de voir dans quelle mesure l'indice grammatical facilite les réponses dans les trois groupes.

Il ressort néanmoins que les anglophones ont nettement plus de facilité que les non anglophones à percevoir la modalité interrogative, ce qui laisse entrevoir que les non anglophones n'exploitent pas tous les indices fournis par l'intonation. En effet, si les caractéristiques de l'intonation présentes dans les stimuli utilisés ici ne diffèrent pas toujours de celles que l'on trouve dans des énoncés semblables en français, il est probable que l'intonation interrogative ne se réalise pas exactement de la même manière dans les deux langues - à propos de l'anglais, voir Armstrong \& Ward (1926) ; Gunlogson (2001) ; Liu \& Xu (2007) ; O'Connor \& Arnold (1961) ; Wells (2006) -, et que les anglophones sont sensibles à cette intonation dès son amorce.

\subsection{Aisance des anglophones dans l'identification des interrogatives non canoniques - exploitation d'indices intonatifs}

41 L'interaction entre groupe et modalité donne à cet égard un résultat particulièrement intéressant: les locuteurs anglophones identifient plus rapidement les énoncés interrogatifs que les énoncés déclaratifs alors que pour les deux groupes d'apprenants c'est l'inverse. On peut faire l'hypothèse qu'en l'absence d'indices grammaticaux, les anglophones sont plus rapides à repérer une intonation interrogative que déclarative parce que les caractéristiques qui signalent un énoncé interrogatif sont décelables plus tôt dans la phrase que celles propres à un énoncé déclaratif, mais qu'il faut une compétence proche de celle d'un anglophone pour repérer ces caractéristiques.

Les énoncés de la première et de la troisième séries ont une syntaxe déclarative par défaut, ce qui permet dès lors de considérer que la modalité interrogative est la modalité marquée : elle seule permet de réinterpréter comme interrogatifs des segments dont la syntaxe serait par défaut déclarative. En d'autres termes, dans ce type d'énoncé, l'intonation interrogative est plus marquée car c'est la seule information permettant de distinguer une modalité qui ne transparait pas dans la syntaxe, mais les apprenants n'arrivent pas encore à exploiter cette information aussi efficacement que les anglophones.

43 Dans la mesure où ces énoncés interrogatifs sont moins fréquents en anglais qu'en français, mais néanmoins suffisamment répandus pour nécessiter un traitement rapide à des fins de communication, les anglophones ont développé cette compétence fine qui n'est pas encore à la portée des apprenants même après un an passé dans un pays anglophone. Ainsi, le transfert positif du français vers l'anglais permet bien de ne pas commettre trop d'erreurs, mais les apprenants ne se sont sans doute pas encore affranchis du besoin d'indices syntaxiques, ce qui leur rend difficile d'exploiter l'information intonative avec autant d'efficacité qu'un anglophone. Une meilleure 
sensibilisation des francophones à l'intonation de l'anglais, ou des séjours plus longs en pays anglophones donneraient sans doute de meilleurs résultats mais les données rapportées ici ne permettent pas d'estimer le temps nécessaire pour atteindre ce résultat.

\section{Divergences entre les résultats obtenus en français et en anglais}

Il est à noter que le résultat de cette étude diffère sur ce point de celui de Faraco \& Cavé (2008), dont les expériences menées sur des énoncés français ont mis en évidence que les apprenants non francophones, tout comme les francophones, avaient un temps de réponse plus court après les énoncés interrogatifs qu'après les déclaratifs. On peut faire l'hypothèse que l'une des raisons de la divergence entre les résultats des deux expériences est la fréquence de ce phénomène en français, puisque dans le langage parlé il est sans doute plus fréquent de former une question bipolaire (fermée, ou totale) de cette manière que par une structure syntaxique réservée à l'interrogation. Comme on l'a vu dans l'introduction, on dira plus facilement « Il pleut ? » que «Pleut-il ?» ou que « Estce qu'il pleut?».

L'anglais, en revanche, aura normalement recours à l'inversion auxiliaire-sujet caractéristique des énoncés interrogatifs canoniques ('Is it raining?'), et la formation des questions fait l'objet d'un enseignement approfondi dès le début de l'apprentissage. En anglais L2, les apprenants sont exposés au phénomène étudié ici moins souvent et plus tard que les non francophones en français L2, ce qui peut se traduire par une sensibilité moindre à l'intonation et une reconnaissance de l'interrogation focalisée sur la syntaxe.

Par ailleurs, Faraco \& Cavé soulignent que l'intonation montante caractéristique d'un énoncé interrogatif en français commence souvent assez tôt dans la phrase, mais peut aussi se trouver seulement sur la dernière syllabe, ce qui oblige un interlocuteur à attendre la fin de l'énoncé pour être sûr qu'il a affaire à un énoncé déclaratif. Bien que ce phénomène d'intonation montante sur la dernière syllabe soit aussi représenté en anglais, il est plus rare.

47 Comme nous l'avons indiqué dans l'introduction, et comme nous l'avons rappelé cidessus, cette façon de formuler des questions est moins neutre en anglais qu'en français, ce qui introduit plus de variabilité dans les contours intonatifs. A titre d'exemple, l'énoncé 'He's out of town' (en tenant compte de tous les résultats, locuteur masculin et locuteur féminin confondus) a donné lieu à des temps de réponse chez les anglophones de $335 \mathrm{~ms}$ pour l'intonation interrogative et de $530 \mathrm{~ms}$ pour l'intonation déclarative. Cette différence de presque $200 \mathrm{~ms}$ en faveur de l'énoncé interrogatif n'est plus que de $140 \mathrm{~ms}$ chez les anglicistes. Chez les non anglicistes la tendance s'inverse avec des réponses plus rapides de 50 ms pour la reconnaissance de l'énoncé déclaratif que pour celle de l'énoncé interrogatif.

Cet énoncé, sous sa forme acoustique interrogative, est en fait à la fois une demande de confirmation et une expression de surprise, ce qui mène à une intonation plus marquée que lors d'une simple demande d'information. Les mêmes remarques s'appliquent à des énoncés comme 'You'll call ?' (le locuteur veut s'assurer que son interlocuteur appellera) ou 'You're all right, Kimberly?' (le locuteur espère que Kimberly va bien mais il a des doutes à ce sujet). Dans la mesure où le recours à ce type de question sans indices grammaticaux en anglais s'accompagne souvent d'intentions communicatives particulières, il est 
probable que les anglophones sont sensibles à l'intonation alors que les francophones, surtout non anglicistes, restent en attente de schémas intonatifs plus standard.

Cette interprétation selon laquelle certains indices intonatifs sont présents avant la dernière syllabe d'un énoncé est confortée par des études sur les caractéristiques acoustiques de questions et d'affirmations se distinguant seulement sur la base de l'intonation. Petrone (2011) signale qu'en italien et en allemand, le contraste entre ces deux types d'énoncé peut être présent dans la région pré-nucléaire de la phrase, bien avant la partie finale. Par ailleurs, Liu \& Xu (2007) ont montré qu'en anglais les intonations commencent à diverger dès la syllabe accentuée du premier mot lexical. On peut donc penser que les locuteurs natifs sont hautement sensibilisés à ce type de phénomène.

\section{Les types d'énoncés}

50 En ce qui concerne les types d'énoncés, on constate que la performance face aux phrases syntaxiquement complètes est presque identique, alors que les phrases syntaxiquement tronquées posent plus de problèmes. Ce résultat vaut pour tous les groupes et montre qu'une syntaxe non conforme représente un obstacle à l'interprétation de ces types d'énoncés. Cet obstacle est plus grand que celui d'une difficulté lexicale : les francophones arrivent à répondre sur la base de l'intonation seule, sans prêter attention au sens de la phrase. Pour les participants anglophones, ce sont les énoncés de la troisième série qui sont les seuls à présenter des difficultés (une fois l'expérience terminée, certains anglophones posaient des questions sur le sens de tel ou tel énoncé de cette série).

Ces remarques sont à considérer en conjonction avec l'interaction entre modalité et type d'énoncés. En effet, tous groupes confondus, seuls les énoncés comportant des difficultés lexicales donnent lieu à une meilleure performance pour les déclaratifs que pour les interrogatifs. Il se peut que la difficulté lexicale, bien que ne posant pas de problème globalement, soit néanmoins un obstacle lorsqu'il s'agit de repérer l'intonation interrogative. Les énoncés déclaratifs de cette série étaient les seuls énoncés à ne pas être mis en contexte au moment de l'enregistrement. De ce fait, bien que sur le plan de la prononciation, ces énoncés soient réalisables sans contexte, il se peut que les locuteurs aient réalisé l'intonation déclarative plus clairement que dans les autres séries. Une étude ciblée permettrait de cerner précisément les effets éventuels de différents types de contexte sur l'intonation d'un même énoncé.

\section{Conclusion}

Cette étude prolonge celle menée par Faraco \& Cavé (2008) sur la perception de l'intonation en français. Bien que différent sur certains points, le protocole expérimental était sensiblement le même et les résultats peuvent être comparés. Nous avons attribué la différence dans l'aptitude à détecter une intonation interrogative chez les apprenants du français et chez les apprenants de l'anglais, $1 /$ à la plus grande fréquence de ce type d'énoncés en français ; 2/ à une moindre sensibilisation des apprenants francophones à cette intonation en anglais ; 3 / à la présence de difficultés lexicales ou syntaxiques. Cette interprétation demande à être confirmée par une étude sur d'autres langues, afin que soit mis en évidence d'une part dans quelle mesure les caractéristiques de la L1 influencent la 
perception de l'intonation en L2, et d'autre part dans quelle mesure les caractéristiques de la L2 constituent un obstacle à cette perception.

Une étude expérimentale complémentaire, sur la perception par des anglophones natifs de l'intonation réalisée par des non anglophones, pourrait apporter un éclairage nouveau sur l'assimilation de l'intonation par des francophones apprenant une langue étrangère. Cette étude a déjà été effectuée sur la perception par des locuteurs russophones natifs de l'intonation interrogative réalisée par des non-russophones (Ullakonoja, 2010).

\section{BIBLIOGRAPHIE}

André, C., Ghio, A., Cavé, C., Teston, B. (2003) Perceval: A computer-driven system for experimentation on auditory and visual perception, Proceedings of the International Congress of Phonetic Sciences, Barcelona: UAB, p. 1421-1424.

Armstrong, L. \& I. Ward (1926) A Handbook of English Intonation, Cambridge: Heffer.

Cavé, C., Teston, B., Ghio, A., Di Cristo, P. (1998) A computer-driven system for assessing speech quality and intelligibility, Acta Acustica, 84, 1, p. 157-161.

Davies, M. (2011) Word frequency data from the Corpus of Contemporary American English (COCA). Téléchargé de http://www.wordfrequency.info le 18 mars, 2011.

Faraco, M. \& C. Cavé (2008) Déclaration ou question ? Étude expérimentale sur la perception de l'intonation par des étrangers apprenant le français, Travaux Interdisciplinaires du Laboratoire Parole et Langage, 27, p. 63-73.

Gunlogson C. (2001) True to Form: Rising and Falling Declaratives as Questions in English. Ph.D. thesis, University of California, Santa Cruz. http://www.ling.rochester.edu/people/gunlogson/ gunlogson.html

Liu, F. \& Y. Xu (2007) Question intonation as affected by word stress and focus in English. Poster présenté au 16ème Congrès International des Sciences Phonétiques, Saarbrücken, 6-10 août 2007.

O'connor, J. \& G. Arnold (1961) Intonation of Colloquial English: a Practical Handbook, London: Longman.

Petrone, C. (2011) Contraintes linguistiques et cognitives sur la variabilité des contours mélodiques. Communication au Laboratoire Parole et Langage, 11 mars 2011.

Ullakonoja, R. (2010) How do native speakers of Russian evaluate YES/NO questions produced by Finnish L2 learners?, http://hdl.handle.net/1911/27603, Rice Working Papers in Linguistics, 2, 2010. Wells, J. C. (2006) English Intonation: An Introduction, Cambridge: Cambridge University Press.

\section{ANNEXES}

\section{Corpus d'énoncés dans leur contexte}


Pour chaque item la version interrogative dans son contexte est donnée d'abord, la version déclarative après. Les participants n'entendaient que la partie en italiques.

$1^{\text {re }}$ série : énoncés syntaxiquement complets, comportant un sujet et un prédicat, mais sans syntaxe interrogative

1.1 Her father was silent for a moment. "Did you know your mother had a job before you girls were born?" he asked abruptly.

"She did?" (SG 255)

"Did Naomi help with the arrangements? I don't think so."

"She did. Not a lot, but she did."

1.2 "We're supposed to meet Sheriff Duffy at the Old Town Grill in the center of

Dahlonega," Sal spoke up.

"You got an address?" (SG 275)

"How are we going to find him?"

"You got an address. Look on the map."

1.3. She grabbed the back of a chair. Her face looked grey.

"You're all right, Kimberly?" (SG 262)

"You've got a job, a family. You're all right, Kimberly. It's Sal I'm worried about."

1.4. [on the telephone]

"Mac..." She should say something. Anything. But for the life of her, she couldn't figure out what.

"Be careful," she whispered.

"Al...says."

"You'll call?" (SG 315)

"OK, let's recap. You'll give him a couple of minutes. You'll call. And then you give it to him straight."

1.5. He usually cooked as little as possible.

"We have vegetables?" (SG)

"What have we got in the fridge?"

"Not much. Some cheese. We have vegetables. That's it."

1.6. "Ten grand? You're serious?"

"I deserve it."

The man laughed abruptly, ruffling the boy's hair.

"You want some extra money, son?" (SG 200)

"You're not going to be able to afford it with what you earn. You want some extra money, son."

1.7. "The kid shot Tommy Mark Evans-“"

"Because he had no choice!"

"He could've just as easily shot you."

"You know what? That doesn't make me feel any better!" (SG 339)

"What do we do when we get there?"

"Look, we've been through this before. You know what. Ask Tim if you're not sure." 
1.8. "He told me what to do. I did it. End of story."

Kimberly frowned, not liking this bit of news.

Duff cleared his throat. "Ma'am, this Mr. Dinchara, he got a real name, a physical address?"

(SG 347)

"Listen, it's not as if we're dealing with a ghost. He got a real name, a physical address.

Understand?"

1.9. “And the packets of driver's licenses, left on Special Agent Martignetti's windshield?" Ginny shrugged. 'Don't ask me. I was just doing what I was told."

"You delivered them?" Sal interrupted. "But why? Ordered by whom?" (SG 347)

"How did they get there? They shouldn't be there."

"What do you mean? They were delivered. You delivered them. Remember?"

1.10 He graduated magna cum laude and got himself a football scholarship to Penn State." "He's out of town?" Kimberly asked in confusion. "Going to college in Pennsylvania?" (SG

108)

"He's out of town." Kimberly said. "Going to college in Pennsylvania."

$2^{\mathrm{e}}$ série : phrases tronquées (difficulté syntaxique)

2.1. "Wanna touch it?" Kimberly asked.

"Yes."

She took Rainie's hand, moved it to her left side, just around the curve. Baby McCormack, engaged in her nightly aerobics, did not disappoint.

"Boy or girl?" Rainie asked. "What do you think?” (SG 219)

"Which do you prefer?"

"Boy or girl. We don't mind. We'll take what come."

2.2. “... we still need to identify and track the killer on our own. Once we know who he is, however, we may be able to identify a spouse or family member who can be of some help to us."

"Socioeconomics?"

"Can't figure him out. Talks white trash, but can also sound very crisp when he wants.

And the SUV is nice-a Limited Edition Toyota FourRunner." (SG 223)

"What's he studying?"

"Socioeconomics. Loves it."

2.3. “To judge by the tape, I'd say Veronica Jones was not his first victim. He's had time to refine his methods. Physically, he's white, five nine or five ten, maybe hundred and seventy pounds. Not big, but lean, wiry. And outdoorsy-hiking boots, jeans, the SUV." "Hunter?"

"In this state, a strong possibility." (SG 222)

"What was he doing before he got here?"

"Hunter. Up in Alaska from what I gather."

2.4. Mac stopped talking, too; the silence stretching long.

"Tough case?"

"You know how it is." (SG 248) 
"You look tired, dear. Had a long day?"

"Yeah. The Osgood murder. Tough case. We're not gonna crack it easily."

2.5. Kimberly shrugged. "But why mess around with call spoofing? We've already met face-to-face. There's nothing in the phone calls she couldn't have told me in person." "shy?"

"Don't think so."

"Scared?" (SG 259)

"How are you feeling?"

"Scared. Never done this before."

2.6. "You're a monster."

"Nah, I'm the man in charge. And you'd better start remembering that. Now get the fuck out and don't bother me again. It's the cops' job to ask questions. It's your job to shut the fuck up. Got it?" (SG 211)

"You're sure you know what you have to do?"

"Yeah. Got it. Loud and clear, don't worry."

2.7. “Working?” asked Rainie, barely suppressing a yawn. “Anything interesting?” (SG 217)

"What are you doing? It's two in the morning!"

"Working. Got to hand in this report first thing tomorrow."

2.8. He held up the first glass vial, now devoid of brackish water. "You're talking a soil very high in metals and minerals. Got a microscope?" (SG 243)

"How are we going to analyse it?"

"Easy. Got a microscope. Look."

2.9. "Wanna touch it?" Kimberly asked.

"Yes." (SG 218)

"What are you trying to do?"

"Wanna touch it. Never been this close before."

2.10. The man backed off, making some calculations of his own. "You heard from her?" he asked abruptly.

“Think she'll do it?" (SG 198)

"I'm not sure she can handle this. What do you reckon?"

"Think she'll do it. She's got what it takes."

\section{$3^{\mathrm{e}}$ série : énoncés comportant des difficultés lexicales}

3.1. "How did he get all that stuff up there, do you think?"

"He used a winch?"

"He used a winch."

3.2. "We didn't mean it. We were messing about, that's all. Nothing serious."

"It was just a lark? Is that what you're saying?"

"It was just a lark"

3.3 "I've just had a call from Jack. They're letting him out."

"He's released on bail? How much are they asking?" 
"He's released on bail."

3.4. "They say they don't want to. Too much trouble, they say."

"They're making a fuss? Tell them they have to, or else."

"They're making a fuss."

3.5. "Ken came round. Said Lorraine was really mad. If he hadn't got out of the way, he'd be in hospital. Or worse."

"She took a swipe at him? With a knife, or what?"

"She took a swipe at him."

3.6. "Sue always looks so immaculate. How does she do it? She's been cooking all afternoon and she looks as if she's off to a photo shoot or something."

"I don't know. She wears an apron?"

"She wears an apron."

3.7. "How did they get so much business?"

"They touted around? That's the only way round here."

"They touted around."

3.8. "John's really pleased. Made over fifty quid on the Underground yesterday."

"He goes busking? I never knew that."

"He goes busking."

3.9. "Where's that carrot I bought? You know, the one with the funny shape?"

"I put it in Bugsy's bowl. Why?"

"It's in the hutch? I wanted to show it to the kids."

"It's in the hutch.

3.10. "Doesn't he like my bag? Why? He reckons it's naff?"

"He reckons it's naff."

\section{NOTES}

1. Remerciements - Nous tenons à remercier ici Carine André et Alain Ghio pour leur aide sur le script utilisé avec le logiciel Perceval ; Martine Faraco et Christian Cavé pour leurs conseils ; les relecteurs anonymes de la revue pour leurs remarques et critiques ; Bernard Bel et Loundou Linganzi pour leur aide lors des enregistrements ; Lynn Hetherington-Blin et Rob Watson pour la lecture des énoncés et pour leur patience lors des enregistrements ; les participants francophones et anglophones, enfin, qui ont accepté de donner de leur temps pour prendre part à cette expérience.

2. Les questions ouvertes (aussi appelées partielles, ou questions en wh) ne sont pas pertinentes ici : même lorsque le mot en wh se trouve in situ, elles ne peuvent s'interpréter comme énoncés déclaratifs. Elles sont donc ipso facto exclues de cette expérience.

3. Lisa Gardner, [2008] 2009, Say Goodbye, New York, Bantam Books. 


\section{RÉSUMÉS}

En anglais comme en français, on peut formuler une question sans changer la syntaxe d'un énoncé, simplement en lui associant une intonation interrogative. Cette étude porte sur la perception de deux modalités d'intonation - interrogative et déclarative - dans des énoncés comportant trois niveaux de difficulté syntaxique ou lexicale. Les participants avaient pour tâche de discerner quelle était la modalité de chaque énoncé, interrogative ou déclarative, et de réagir à l'aide d'un dispositif enregistrant leur temps de réponse. Il y avait trois groupes de participants : des anglophones, des étudiants français anglicistes ayant séjourné une année en pays de langue anglaise et des étudiants français non anglicistes. Les résultats permettent de dégager des tendances par groupe: les anglophones répondent plus rapidement que les anglicistes qui sont eux-mêmes plus rapides que les non anglicistes. Il y a également une interaction entre groupes et modalité, car les étudiants francophones identifient les énoncés déclaratifs plus rapidement que les interrogatifs, alors que pour les anglophones c'est l'inverse. En ce qui concerne les types d'énoncés, les temps de réponse sont significativement plus lents pour les phrases syntaxiquement incomplètes.

In English, as in French, a question can be formed without changing the syntax of a sentence but simply by giving it an interrogative intonation. This study examines the perception of two intonation modalities, interrogative and declarative, in spoken sentences which included three levels of syntactic or lexical difficulty. The participants' task was to discern the modality of each sentence, interrogative or declarative, and to react by means of a device which recorded their response times. There were three groups of participants: native English speakers, French students of English who had lived in an English-speaking country for a year and French students studying a subject other than English. Results reveal a significant group factor with native speakers responding more quickly than specialist students of English who responded quicker than the non-specialists. There was also an interaction between group and modality, whereby French speakers identified declaratives more quickly than interrogatives, but the opposite held for the native speakers. Regarding the type of sentence, response times were significantly slower for syntactically incomplete sentences.

\section{INDEX}

Mots-clés : perception, intonation, énoncés déclaratifs/interrogatifs, questions bipolaires, anglophones et francophones

Keywords : declarative, interrogative, yes-no questions, native and non-native speakers

\section{AUTEURS}

\section{JEAN-MARIE MERLE}

Université de Nice Sophia Antipolis

Jean-Marie.MERLE@unice.fr 


\section{PETER PRINCE}

Aix-Marseille-Université - LPL- CNRS UMR 7309

Peter.Prince@lpl-aix.fr 